\title{
OPTIMIZING DIRECT PRINTING OF PZT THIN FILMS
}

\author{
S. Bathurst ${ }^{l}$, J. Jeon ${ }^{l}$, P. Mardilovich', H.W. Lee ${ }^{l}$, and S.G. Kim ${ }^{l}$ \\ ${ }^{1}$ Massachusetts Institute of Technology, Cambridge, Massachusetts, USA \\ ${ }^{2}$ Hewlett Packard, Corvallis, Oregon, USA
}

\begin{abstract}
We recently reported a new method for depositing lead zirconate titanate (PZT) thin films via thermal ink jet (TIJ) printing of a modified sol-gel [1]. The use of PZT in MEMS has been limited due to the lack of process compatibility with existing MEMS manufacturing processes. Direct printing of PZT thin films eliminates the need for photolithographic patterning and etching, allows for controlled deposition over non-planar topographies, and enables the fabrication of devices with varying thickness which cannot be accomplished with conventional spin coating processes. This paper reports conditions of deposition and crystallization for PZT thin films via thermal ink jet printing. Included are details of the solution chemistry developed, printing conditions required for MEMS quality films, and thermal processing parameters that enable a strong piezoelectric response.
\end{abstract}

\section{INTRODUCTION}

The high degree of piezoelectric and ferroelectric coupling in perovskite phase lead zirconate titanate (PZT) makes it an attractive material for use in MEMS. Due to the strong piezoelectric response and low operating voltage, many groups have worked to integrate thin film PZT into a wide range MEMS devices including: actuators, energy harvesters, resonators, pressure sensors, pumps, nano-positioning stages, and MEMS switches [2]-[4]. However, processing of thin film PZT is not readily compatible with existing MEMS fabrication processes and significant design constraints exist when integrating thin film PZT.

While some recent work has demonstrated novel ways of forming sol-gel based films, spin coating remains the dominant method of forming thin film PZT for MEMS. Not only is spin coating inherently wasteful of the expensive and difficult to manufacture sol, but it prevents the deposition of PZT films on or around out of plane features. Spin coating is not the best fabrication method for PZT based MEMS, but it is the most compatible at the present time. Figure 1 shows the cracking and thinning that occurs when PZT is spin coated over a step about $2 \mu \mathrm{m}$ in height. The sol is also very sensitive to other deposition parameters, including humidity, particle contamination, and substrate condition and as a result device yields are often low. Reliable deposition requires great care and often a good deal of experience processing PZT thin films. Consequently a flexible new approach is needed to easily and effectively deposit high quality PZT thin films in MEMS applications.

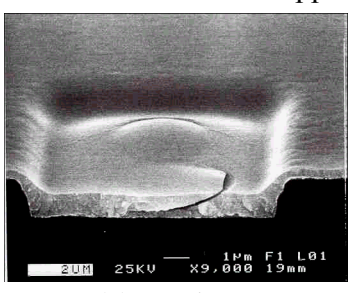

(a) cracking

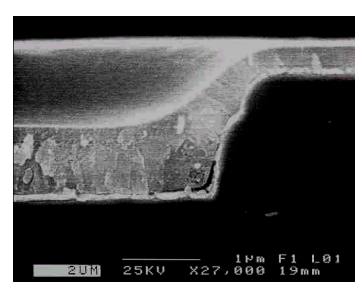

(b) thinning \& high stress
Figure 1: SEM images of poor step coverage of spin coated solgel PZT films [5].

In recent years dot-on-demand (DOD) printing has been studied as a robust, flexible, and inexpensive method of material deposition for MEMS [6]. Some of the benefits of DOD printing for MEMS can be summarized as follows. First, no mask or patterning is required. Direct printing enables the designer to deposit a film based on a digital pattern file only. This file can be generated in many ways, including from computer aided design (CAD) software, manually, or based on images. Digital deposition in this way eliminates the need for photolithography and subsequent etching steps in the manufacturing process flow. Furthermore the short cycle time required for pattern generation makes rapid prototyping possible and allows multiple design iterations that were previously not possible in MEMS product development. A further advantage of direct printing is the cost savings due to a reduction in the material consumption during manufacturing and in chemical waste produced. The result is a manufacturing process that is both cleaner and cheaper than other common deposition techniques.

Perhaps the most compelling benefit of direct printing of PZT is that it provides a freedom of geometry that eliminates many of the design constraints currently associated with PZT MEMS. Since high quality thin films can be achieved with deposition control that is not possible with spin coating, novel functionalities can be incorporated into PZT MEMS. Specifically, PZT printing is able to deposit material over and around large out-of-plane features. In addition, the thickness of thin film PZT can vary deterministically across a device or across a wafer. Thickness can be controlled from tens of nanometers for sol-gel based inks, all the way to very high aspect ratio features $100 \mu \mathrm{m}$ tall or thicker with particle based inks [7]. Together, these improvements enable a new geometry of device designs that were previously not possible with simple manufacturing processes. While it is unclear yet what novel devices this new manufacturing method will yield, it is possible that the precise deposition control achieved with PZT printing could have a significant impact on the way MEMS structures are designed in the future.

In order to achieve the potential benefit of direct printing of PZT, three process requirements must be addressed. First, the ink must print reliably, forming discrete drops without clogging. Second, the geometry of the printed film must be highly uniform and controllable. Finally the properties of the printed PZT film, both mechanical and electrical, must be comparable to those of the spin coated PZT film.

\section{SOLUTION CHEMISTRY}

The sol-gel used in this work was Mitsubshi A6 50/49 PZT. As purchased, this sol is $85 \%$ (wt) 2-methoxyethanol and $15 \%$ (wt) a mixture of lead, zirconium, and titanium oxides. Dilution is required to control the evaporation rate of the ink and prevent decomposition of the metal-organic molecules on the ink jet heater. Combinations of 2-methoxyethanol, isopropanol, and 2ethylhexanoic acid were added to the sol to created the diluted PZT inks. 2-methoxyethanol is the same solvent used in the manufacture of the PZT sol and helps to control hydrolysis. 2propanol is a common mild solvent with a low boiling point that is known to be reliable in thermal inkjet printing. Finally 2ethylhexanoic acid was used to control overall ink volatility as it has a high boiling point and is also known to be compatible with thermal ink jet head.

To prevent clogging of the printer nozzle and defects in the 
film, three sources of particle contamination were addressed and controlled. First, to eliminate external particle contamination each ink was filtered with a $0.45 \mu \mathrm{m}$ PTFE syringe filter and deposition was carried out in a hermitically sealed glove box filtered with $99.99 \%$ efficient removal of 0.2 micron and larger airborne particles. Particle formation during the printing process was also a concern due to the decomposition of the metal-organic molecules during the thermal event. Throughout this work, over thirty ink chemistries, with dilution levels ranging from the as purchased $15 \% \mathrm{wt}$ of metal oxides down to $2 \% \mathrm{wt}$, were tried and empirically the appropriate levels of dilution were observed. Table 1 shows observations of the concentration required for reliable printing for different nozzle sizes. Finally, preventing clogging also requires controlling the evaporation rate of the ink such that a stable meniscus is formed at the nozzle. If the solvent evaporates too quickly, metal oxide particles are built up inside the nozzle and firing chamber and concentrations that exceed stable printing requirements result. This was prevented by maintaining a low rate ink flow through the nozzle at all times.

Table 1: Maximum allowable metal oxide concentrations for reliable printing with different droplet sizes.

\begin{tabular}{|c|c|}
\hline $\begin{array}{c}\text { Nozzle size, droplet } \\
\text { volume (pl) }\end{array}$ & $\begin{array}{c}\text { Maximum Acceptable } \\
\text { Concentration (\%wt) }\end{array}$ \\
\hline 180 & 15 \\
\hline 80 & 4.7 \\
\hline 35 & 2.3 \\
\hline
\end{tabular}

To ensure predictable substrate wetting and stable droplet formation, certain dimensionless numbers known to govern drop on demand printing dynamics were calculated and observations were made to ensure the accuracy of the predicted results. The forming of a film on a substrate is characterized by the Bond number, which for these droplet sizes will be no larger than approximately $4 \cdot 10^{-3}$. As for most ink jet printing, the Bond number for this work is sufficiently low to ensure the film shape on the surface will be dominated by surface tension, forming spherical caps for single drops and cylindrical slices for a printed line. Figure 2 shows an image of a spherical cap of 2-methoxyethanol forming on a platinum substrate during contact angle measurements. The contact angle was measured between $10^{\circ}-12^{\circ}$, although accuracy can not be guaranteed for measurements at such low angles. Previous work has shown that the ratio of the Reynolds number to the square root of the Weber number dictates the dynamics associated with droplet formation [8]. Most dot-ondemand inks have $1<Z<10$. Viscous dissipation can prevent droplet ejection for $Z<1$ and for $Z>10$ multiple drops, or even a constant stream, can result. $Z$ is estimated to be 1-3 for the smaller nozzles sizes used in this work.

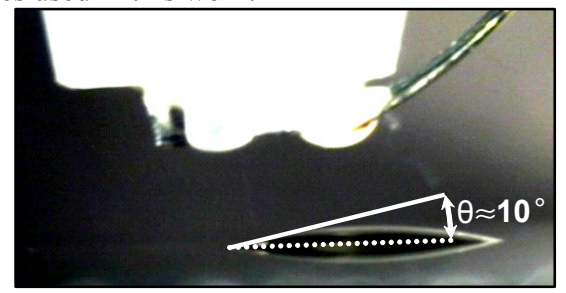

Figure 2. Advancing contact angle measurement of 2methoxyethanol on platinum.

\section{CONTROLING FILM GEOMETRY}

This work characterized the resolution limits of two methods of printed PZT patterning. The first involved printing into a predefined mold and the second, free printing method, was based only on droplet size and wetting angle with the substrate. Printing into a mold to pattern the PZT removes the coupling between pattern resolution and film uniformity that occurs due to the dependence both parameters have on film evaporation rate. When using a mold, droplet spreading and solute diffusion can be independently controlled. Therefore the evaporation rate can be very low, allowing for highly uniform films without a loss of resolution. Figure 3 demonstrates the resolution achieved for cast PZT films. In general, the in plane geometry PZT films printed into lithographically pattern molds was controlled to the accuracy of the mold itself. Thickness uniformity was also good: for printed films with a bulk mean thickness of $147 \mathrm{~nm}$, the RMS roughness was $9 \mathrm{~nm}$.
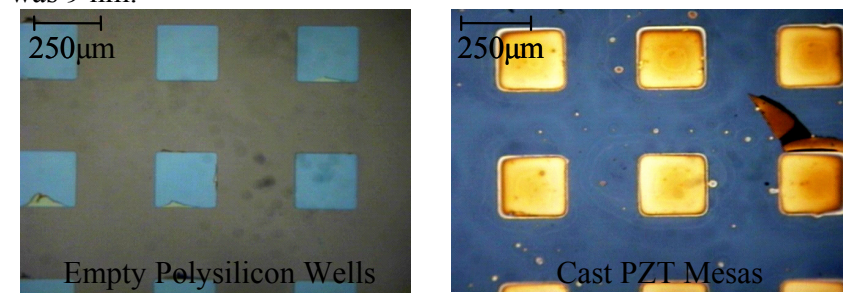

Figure 3. Results of thermal ink jet printing of PZT into preformed polysilicon molds.

Mold-free printing for MEMS is attractive because the mold removal step after printing adds complexity to the process. However, printing without a mold makes it more difficult to achieve the geometric control required. The diffusion of solutes towards the film edges during solvent evaporation known as the coffee stain effect can lead to significant non-uniformity []. Figure 4 shows how early printed PZT films exhibited this effect. In order to overcome this non-uniformity a study was conducted to determine the ink volatility and substrate temperature required to achieve the optimum level of spreading and diffusion.

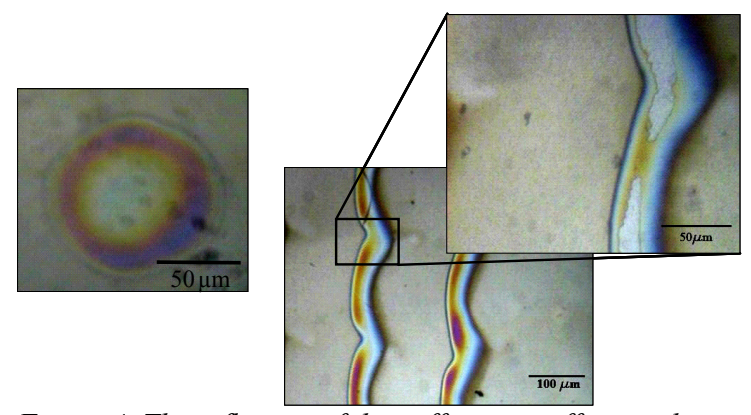

Figure 4. The influence of the coffee stain effect on dot on demand printing of $P Z T$

Controlling the thickness uniformity of free printed PZT films requires controlling the amount of diffusion of the metal oxides towards the film edges that can occur as the volatile solvents evaporate. It was observed that ink chemistries with an excess of 2methoxyethanol always exhibited significant metal oxide diffusion flow, and therefore substantial non-uniformity. Given the relatively high levels of dilution required, isopropanol was selected to make up the bulk of the ink given that it had a lower boiling point and would evaporate more quickly controlling diffusion of the oxides. It was found that inks that were made up of 50\% isopropanol and $15 \%$ PZT sol would both print reliably as required by the clogging constraints, and evaporate quickly enough to prevent significant solute diffusion.

To achieve highly uniform films, precise control over evaporation rate is required. This was accomplished by adjusting 
the substrate temperature. However, the range of substrate temperatures available is limited to approximately $70^{\circ} \mathrm{C}$ to prevent clogging as a result evaporation of ink from the printer nozzle. Due to this limit, the volatility of the ink was controlled to bring the substrate temperature into the specified range. This was accomplished using the remaining $35 \%$ of the ink which was comprised of a mixture 2-methoxyethanol and 2-ethylhexanoic acid. The boiling point of ethylhexanoic acid is significantly higher than the other solvents $\left(228^{\circ} \mathrm{C}\right)$, and therefore the concentration of ethylhexanoic acid was used to control the overall ink volatility, with anhydrous 2-methoxyethanol making up the remainder of the ink. Figure 5 demonstrates the control over mold free film geometry that can be achieved by adjusting the substrate temperature when the ink volatility is set at an acceptable level.
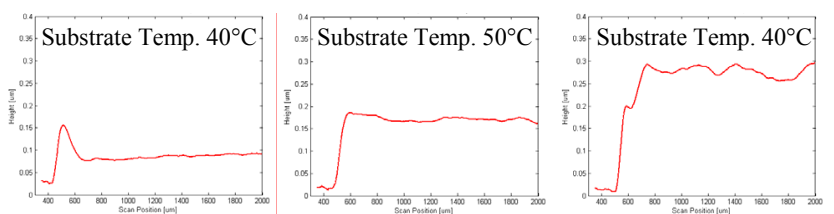

Figure 5. Profilometry of thermal ink jetted PZT, deposited at different substrate temperatures. Ink composition: $6 \%$ EHA, $15 \%$ PZT sol-gel, 50\%IPA, 29\%ME.

In order to determine the optimal substrate temperature for this ink (6\% EHA, 15\%PZT, 50\%IPA, 29\%ME), 15 samples were prepared at different temperatures. The results of the study clearly showed an optimum deposition temperature at $60^{\circ} \mathrm{C}$. However, to ensure that clogging of the nozzle due to excessive solvent evaporation would not be a problem, it is desirable to reduce the substrate temperature during deposition. A new ink was prepared made up of 5\% EHA, 15\%PZT, 50\%IPA, 30\%ME, and the study was conducted again. The results of the second uniformity vs. substrate temperature study show a reduction in optimum deposition temperature from $60^{\circ} \mathrm{C}$ to $50^{\circ} \mathrm{C}$ (Figure 6). These two studies demonstrate the effectiveness of using substrate temperature to control deposition uniformity, and using ink volatility to ensure that the substrate temperature doesn't cause clogging.

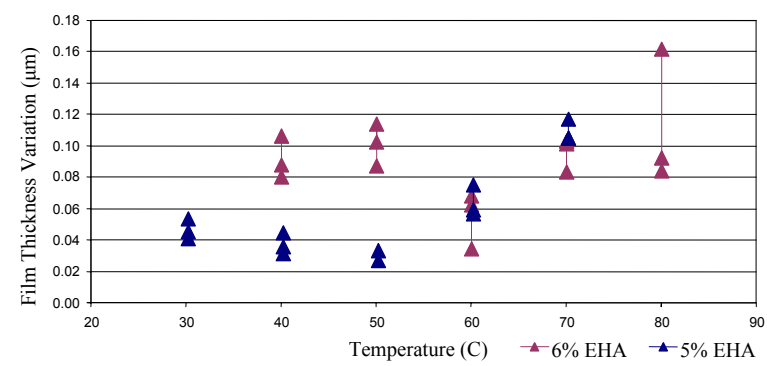

Figure 6: Study of thickness variation vs. substrate temperature for thermal ink jetted PZT on Pt.

After optimization of solution chemistry and substrate temperature, PZT films of between $100-500 \mathrm{~nm}$ thickness with less than $40 \mathrm{~nm}$ variation could be printed with a droplet size of 80pl. Spot sizes as small as $43 \mu \mathrm{m}$ were achieved with a $10 \mathrm{pl}$ droplet of PZT ink deposited on Pt substrate. The edge variation of printed lines was controlled within $+/-10 \mu \mathrm{m}$.

\section{THERMAL PROCESSING OF PRINTED PZT FILMS}

There are three steps to properly crystallize a sol gel based PZT thin film into a piezoelectric, perovskite phase. The first is the drying of the solvent in which the metal organics are dispersed. The second is the decomposition of the metal organics into an amorphous film. Finally the film must be annealed into a perovskite structure. The thermal processing of the early printed PZT films was determined based on manufacturer's recommendation and authors' experience in processing spin coated films of the Mitsubshi A-6 50/49 PZT sol gel (Table 2). With the same thermal treatment, the printed PZT showed very poor piezoelectric performance and low film resistivity. At $5 \mathrm{~V}$ the film resistivity measured was $4.5 \cdot 10^{10} \Omega \cdot \mathrm{cm}$, however at $15 \mathrm{~V}$ the resistivity dropped to $8.3 \cdot 10^{2} \Omega \cdot \mathrm{cm}$

Table 2: Thermal processing conditions for spin coated PZT films.

\begin{tabular}{|l|c|c|}
\hline Processing Step & Temp. $\left[{ }^{\circ} \mathbf{C}\right]$ & Time $[\mathbf{m i n}]$ \\
\hline Dry & 260 & 3 \\
\hline Pyrolysis & 360 & 8 \\
\hline Anneal & 650 & 20 \\
\hline
\end{tabular}

In order to improve piezoelectric performance, the drying and pyrolysis steps were lengthened to ensure complete evaporation of the solvent and removal of added organics prior to annealing. FTIR analysis after 2 hours of pyrolysis showed the removal of a significant fraction of the organic material (Figure 7).

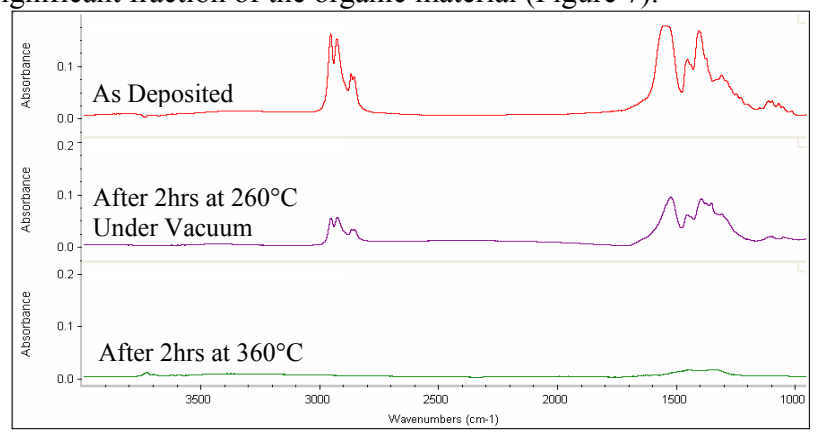

Figure 7: FTIR of a printed PZT thin film during heat treatment.

Under this new thermal treatment the dielectric properties of printed films were significantly increased. At $5 \mathrm{~V}$ the film resistivity measured was $6.4 \cdot 10^{10} \Omega \bullet \mathrm{cm}$, and at $15 \mathrm{~V}$ the resistivity was still $6.2 \cdot 10^{10} \Omega \cdot \mathrm{cm}$.

$\mathrm{X}$-ray diffraction was performed after annealing to investigate the influence of pyrolysis time on crystallization. All films had a pure perovskite, pyroclore free, crystal phase. The study further showed that the amount of crystalline PZT increase significantly with pyrolysis time.

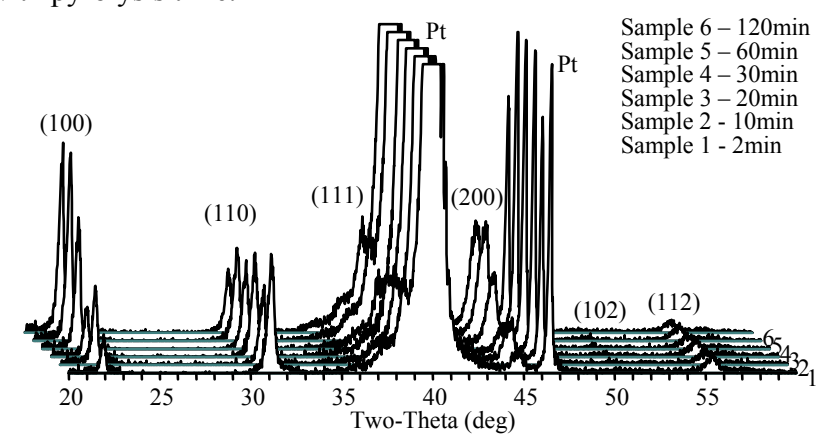

Figure 8: X ray diffraction of printed PZT films pyrolised for different times.

The substantial improvement of the polarization-voltage curve of the extended pyrolysis is shown in Figure 9. When compared to 
a spin coated film, the remnant polarization of the extended pyrolysis film (approx. $+/-5 \mu \mathrm{C} / \mathrm{cm}^{2}$ ) is still lower than desired, but shows much improved dielectric performance and a piezoelectric performance that is still greater other piezoelectric materials. The test device fabricated was a simple capacitor structure with approximately $400 \mathrm{~nm}$ thick printed PZT between two platinum electrodes. The bottom electrode on which the PZT was printed was platinum $(200 \mathrm{~nm} \mathrm{Pt} / 20 \mathrm{~nm} \mathrm{Ti} / 200 \mathrm{~nm} \mathrm{SiO} 2$ / Si). The capacitor area was $6.25 \cdot 10^{-4} \mathrm{~cm}^{2}$.

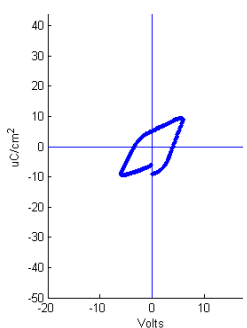

(a)

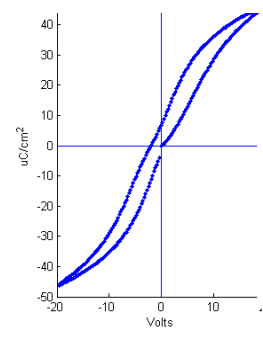

(b)

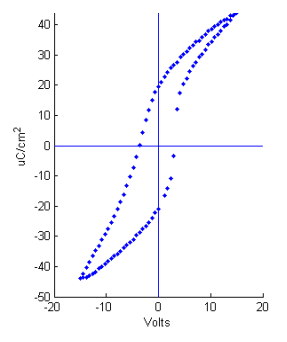

(c)
Figure 9: Polarization vs. voltage hysteresis curve for a thermal ink jetted PZT thin film a) after standard pyrolysis and b) after extended pyrolysis as well as c) for a spin coated film after standard pyrolysis.

It is believed that the relatively low remnant polarization is due to defects/voids forming at the grain boundaries (Figure 10). These defects are likely due to incomplete removal of organics before annealing, which were added when the sol was dilute to become ink jettable. This theory is supported by the FTIR spectrum in figure 7 showing some organics remaining after pyrolysis, as well as the increase in crystallinity with increased pyrolysis indicated by the x-ray diffraction in figure 8 . Furthermore, thermal gravimetric analysis, not shown, indicates that increasing the ramp rate of the pyrolysis leads to a greater organic removal. Work to optimize pyrolysis conditions to eliminate these defects is underway.

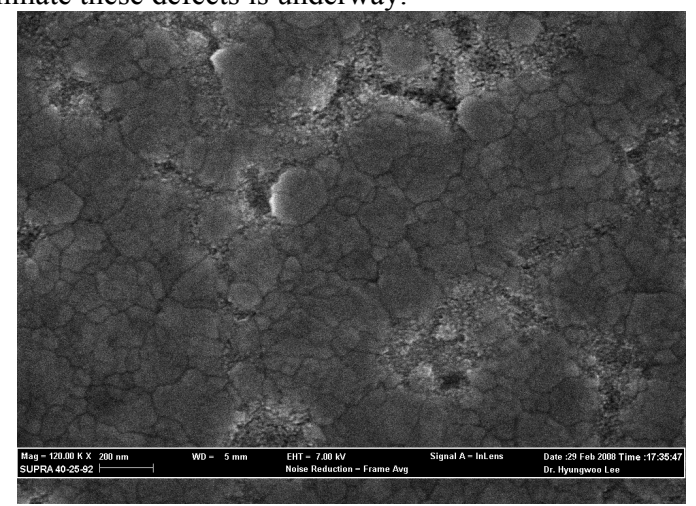

Figure 10: SEM image of printed PZT film showing intergranular defects.

\section{CONCLUSION}

A new solution based, dot-on-demand (DOD) PZT deposition method has been developed that provides increased flexibility and lower manufacturing costs over standard deposition methods. Ink chemistry that can be reliably ink jetted has been developed and optimized; printing conditions are optimally determined; the appropriate thermal processing parameters are identified and characterized to ensure a high resistivity and good perovskite phase of crystallized PZT films after annealing. This required implementing particle control measures, determining the right amount of dilution required to control droplet formation, and ensuring minimal evaporation at the printer nozzle.

Analysis was performed on droplet formation dynamics and on substrate wetting conditions. A method for determining the optimum deposition temperature for dot on demand printing of highly uniform thin films on a nonporous surface was presented. Furthermore, a method for creating and using cast features as mold for thin film deposition was developed. Printing of cast films was demonstrated to have significantly improved resolution and uniformity over standard drop on demand deposited films.

Finally the thermal processing conditions were determined for the annealing of printed PZT films into pure perovskite phase poly crystalline films. Pyrolysis time was investigated as a means for controlling film crystallization. Fourier transform infrared spectroscopy was used to confirm sufficient removal of the organic material in our highly diluted PZT ink and X-ray diffraction test was used to confirm the final crystalline phase.

The results from this study will provide a robust and efficient method of depositing PZT thin films for MEMS applications. This will also enable many novel MEMS device designs that were not previously possible.

\section{AKNOWLEDGEMENTS}

This study was supported by the DARPA Grant HR0011-061-0045 and Hewlett Packard. The authors would like to thank Paul Benning and Murali Chaparala of HP for their contributions and help.

\section{REFERENCES}

[1] S. Bathurst, H.W. Lee and S.G. Kim, "Direct Printing of Lead Zirconate Titanate Thin Films," IEEE MEMS 2008, Tucson, AZ, 2008

[2] P. Muralt, "PZT Thin Films for Microsensors and Actuators: Where Do We Stand?" Ultrasonics, IEEE Transactions on Ferroelectrics and Frequency Control, vol. 47, pp. 903-915, July 2000 .

[3] Z. Traina and S.G. Kim, " A Large Strain Piezoelectric Microactuator by Folding Assembly," M.S. thesis, Massachusetts Institute of Technology, Cambridge, MA, 2005.

[4] R. Sood and S.G. Kim, "Piezoelectric Micro Power Generator (PMPG): A MEMS-Based Energy Scavenger," M. Eng. thesis, Massachusetts Institute of Technology, Cambridge, MA, 2003.

[5] S.G. Kim, M.K. Koo, "Design of a microactuator array against the coupled nature of microelectromechanical systems (MEMS) processes", in Annals of the CIRP, vol. 49, no. 1, 2000

[6] S. B. Fuller, E. J. Wilhelm, and J. M. Jacobson, "Ink-Jet Printed Nanoparticle Microelectromechanical Systems," Journal of Microelectromechanical Systems, vol. 11, no. 1, February 2002

[7] R. Noguera, C. Dossou-Yovo, M. Jejeune, and T. Chartier, "Fabrication of 3D fine scale PZT components by ink-jet prototyping process," Journal de Physique IV, vol. 128, pp. 87-93, 2005

[8] J.E. Fromm, IBM J. Res. Dev. 28 (1984) p. 322.

[9] R.D. Deegan, O. Bakajin, T.F. Dupont, G. Huber, S. R. Nagel, T.A. Witten, "Capillary flow as the cause of ring stains from dried liquid drops", Letters to Nature, vol 389, no. 6653, pp 827-829, 1997 